\title{
Análise Dinâmica do Comportamento de Filas de Mensagens para o Aumento do Paralelismo de Consumo
}

\author{
Eduardo Henrique Teixeira ${ }^{1}$, Aletéia Patrícia Favacho de Araújo ${ }^{1}$ \\ ${ }^{1}$ Departamento de Ciência da Computação - CIC \\ Universidade de Brasília (UnB) - Brasília, DF - Brasil \\ edu.henr@gmail.com, aleteia@cic.unb.br
}

\begin{abstract}
Resumo. A elasticidade em computação consiste em dimensionar adequadamente os recursos necessários para processar uma aplicação distribuída. Para isso, são necessários mecanismos para evitar o fenômeno do limiar de detecção de elasticidade para cima ou para baixo. Este artigo propõe um middleware para analisar dinamicamente os fluxos de filas de mensagens, e um mecanismo para aumentar o paralelismo de consumo baseado no comportamento da vazão. Dessa forma, é apresentada a arquitetura do middleware IOD (Increase On Demand) com suporte ao aumento e a diminuição de threads, para conter o crescimento de filas de mensagens, utilizando a técnica de heurísticas baseada em limites por um determinado tempo, e o agrupamento de mensagens em subfilas de acordo com um critério de classificação.
\end{abstract}

\section{Introdução}

Elasticidade é a característica de um ambiente que define o grau no qual um sistema é capaz de adaptar-se dinamicamente às mudanças de carga de trabalho, por meio do provisionamento e da liberação de recursos de forma automática [Herbst et al. 2013]. Assim, computação elástica é o provisionamento dinâmico de recursos [Perez et al. 2009].

A computação elástica traz enormes vantagens para os provedores de aplicações, incluindo economia de custos e prevenção de super e sub-provisionamento de recursos de TI. Isso ocorre por meio do monitoramento da demanda e da aquisição dos recursos requeridos pelas aplicações para alcançar um alto nível de qualidade [Leitner et al. 2012].

O objetivo da elasticidade é que a quantidade de recursos alocada para um serviço seja a que ele realmente necessita. Dessa forma, é possível, por exemplo, reduzir o número de servidores necessários para processar as filas de mensagens de um sistema distribuído e, consequentemente, economizar recursos computacionais. Também é possível determinar a carga atual de processamento requerida em um cluster, de acordo com a demanda exigida, que pode ser medida em função da vazão de saída em uma fila de mensagens, e que pode ser limitada pelo consumo de CPU, para evitar a saturação dos servidores. Para este fim, em uma plataforma distribuída, é necessário um middleware, que seja capaz de implementar, de maneira transparente e dinâmica, o conceito de elasticidade, a fim de garantir que o processamento possa se adaptar ao crescimento das filas de mensagens, evitando que as mensagens se acumulem antes de serem processadas.

Por outro lado, em plataformas distribuídas, além da elasticidade, é fundamental garantir tolerância a falhas, pois, dessa forma, um ou mais processos podem falhar sem prejudicar o restante do sistema [Tanenbaum and Steen 2008]. Falhas são frequentes, e 
podem ocorrer devido a erros de hardware ou software [Smith 1986]. As falhas de hardware resultam, geralmente, da degradação física de componentes, enquanto que as falhas de software ocorrem devido a erros no projeto ou na implementação, e são também conhecidas como bugs [Smith 1986]. Nesse contexto, a tolerância a falhas de software é a garantia do comportamento correto da aplicação, independente do número e do tipo de falhas que ocorram [Coulouris et al. 2009].

Assim sendo, este artigo propõe o middleware IOD (Increase On Demand) que garante elasticidade e tolerância a falhas de software em arquiteturas de sistemas distribuídos baseadas em filas de mensagens, como são os clusters de alto desempenho e de alta disponibilidade. O middleware IOD analisa dinamicamente o comportamento da vazão das filas para determinar a necessidade de aumentar ou de diminuir o número de threads responsáveis pelo tratamento das mensagens. O objetivo do middleware proposto é evitar o enfileiramento das mensagens a serem processadas. O IOD também analisa o comportamento do consumo de CPU para determinar os limites de escalabilidade dos servidores para evitar a saturação do cluster. Além disso, ele utiliza mecanismos para recuperação rápida em caso de falhas, garantindo que os serviços disponibilizados pelo cluster sejam disponibilizados rapidamente.

Dessa forma, o foco do middleware IOD é utilizar a computação elástica para que os núcleos das unidades de processamento, disponíveis no cluster, sejam utilizados mais eficientemente. Para isso, no middleware proposto, a demanda necessária de threads para tratar as filas de mensagens é calculada, dinamicamente, de acordo com o comportamento da vazão média de saída e do consumo médio de CPU de cada nó computacional. Dessa forma, as aplicações distribuídas que utilizam filas de mensagens como mecanismo de IPC (Inter Process Communication) [Gray 2003], podem se beneficiar das técnicas projetadas no middleware IOD, proposto neste artigo. Isso é possível porque o dimensionamento da utilização dos recursos disponíveis no cluster é realizada em cada nó, evitando períodos de ociosidade de CPU.

Para apresentar o middleware IOD, o restante deste artigo está dividido em mais 4 seções. A Seção 2 contém uma visão geral sobre trabalhos relacionados à elasticidade e à tolerância a falhas. A Seção 3 descreve a arquitetura do middleware proposto IOD. Em seguida, a Seção 4 apresenta a avaliação e a análise dos testes realizados com o middleware IOD. Para finalizar, a Seção 5 apresenta algumas conclusões e descreve os próximos passos deste trabalho.

\section{Trabalhos Relacionados}

Para alcançar a elasticidade de forma transparente e automática, as abordagens citadas em [Tran et al. 2011][Ma et al. 2010] são adequadas quando a integridade sequencial no processamento de mensagens das filas não é um requisito funcional. Assim, as mensagens nas filas podem ser redistribuídas de acordo com a vazão requerida, sem a preocupação com a ordem em que elas são processadas. Entretanto, isso pode ser uma desvantagem quando há a necessidade de manter a integridade sequencial baseada na ordem de entrega para os consumidores das filas. Nesse caso, os eventos gerados pelos consumidores precisam respeitar a restrição de tempo na qual as mensagens foram geradas.

As atribuições feitas pelos publishers aos subscribers podem gerar assimetrias de distribuição dos dados [Tran et al. 2011][Li et al. 2011][Fang et al. 2011]. Essas assi- 
metrias também são comuns em sistemas com múltiplas filas de mensagens e múltiplos consumidores [Tran et al. 2011][Li et al. 2011]. Identificar os melhores candidatos para o consumo das filas é necessário para reduzir a latência e evitar a saturação de consumidores sobrecarregados. Assim, é possível determinar esses candidatos identificando a maior vazão média [Tran et al. 2011][Li et al. 2011]. O menor consumo médio de CPU também pode ser utilizado como métrica de identificação dos melhores candidatos para o consumo de novos itens [Li et al. 2011].

é possível $\begin{array}{ccc}\text { Em } \\ \text { explorar a transparência de elasticidade }\end{array}$ para a aplicação [Tran et al. 2011][Ma et al. 2010][Imai et al. 2012]. Segundo [Li et al. 2011][Imai et al. 2012][Sugiki and Kato 2011], essa transparência pode ser alcançada utilizando a métrica de carga exigida em função do consumo de CPU. Entretanto, se o número de mensagens permanecer próximo do limite de detecção de elasticidade, problemas de início e término rápido de threads podem ocorrer. Para sanar esse problema e manter a vazão em níveis aceitáveis, [Tran et al. 2011][Li et al. 2011][Imai et al. 2012] propõem o uso de heurísticas com abordagem baseada em limites, por um determinado tempo. Dessa forma, as threads criadas são mantidas por mais tempo, o que minimiza o custo de reinício e diminui a latência do consumo de mensagens. Essa abordagem é interessante para sanar o problema do limiar de detecção de elasticidade para cima ou para baixo.

As abordagens [Guo et al. 2012][Imai et al. 2012][Leitner et al. 2012] [Ma et al. 2010][Sugiki and Kato 2011][Tran et al. 2011] citam que a elasticidade deve ser alcançada de forma transparente e automática. Também foi abordado o uso do padrão de projeto publisher/subscriber [Fang et al. 2011][Li et al. 2011][Tran et al. 2011] para alcançar a elasticidade e o desacoplamento de produtores e consumidores. Nas abordagens citadas em [Imai et al. 2012][Li et al. 2011][Sugiki and Kato 2011][Leitner et al. 2012] a elasticidade é alcançada de acordo com a demanda de carga exigida, que é medida pelo consumo de CPU. No entanto, em todas as abordagens estudadas não há uma preocupação com a integridade sequencial com que as mensagens assíncronas são processadas, nem com as assimetrias de distribuição dos dados para alcançar o paralelismo de consumo.

Muitas das abordagens de tolerância a falhas utilizam a replicação para que outro conjunto de processos assuma o processamento em caso de queda ou falha [Abbes et al. 2010][Bicer et al. 2010][He et al. 2012][Martins et al. 2010]. No entanto, técnicas como a recuperação rápida de falhas [Bicer et al. 2010][Castro et al. 2012][He et al. 2012][Wang et al. 2009] são interessantes, pois evitam os custos de memória adicional e de comunicação entre os processos replicados para a troca das informações de estados. [Castro et al. 2012][Martins et al. 2010] propõem o uso do suporte à tolerância a falhas de forma transparente no middleware. Essa abordagem é atrativa, pois abstrai da aplicação usuária os detalhes de detecção e de recuperação das características implementadas. Para alcançar essa transparência [Bicer et al. 2010][Martins et al. 2010] utilizam a detecção da queda ou falha por meio do monitoramento da conexão entre os processos. 


\section{Middleware Proposto}

Para criar ambientes elásticos eficientes, os serviços existentes devem ser estendidos com funcionalidades de computação elástica e com políticas de provisionamento de recursos sob demanda [Marshall et al. 2012]. Dessa forma, o middleware IOD propõe o suporte à elasticidade por meio da adaptação dinâmica do número de threads para tratamento das mensagens baseado na análise da vazão de entrada e de saída das filas e do consumo de CPU do servidor. O suporte a tolerância a falhas dos processos, no middleware IOD, é realizado por meio da detecção da queda e da recuperação com reinício rápido. Assim, as mensagens das filas continuam a ser processadas a partir do ponto em que ocorreu a falha, sem a necessidade de uso da replicação de processos. Essas características serão apresentadas nas próximas seções.

\subsection{Arquitetura da Estrutura de Dados}

Para alcançar o paralelismo de consumo, é necessário, primeiramente, separar as mensagens de uma fila em grupos distintos, de acordo com um critério de classificação. Assim sendo, cada fila dá origem a várias subfilas, cada uma indexada por uma chave que identifica um grupo. Isto deve ser feito para aumentar o número de worker threads que serão responsáveis por processar as mensagens, distribuindo os grupos entre as várias worker threads iniciadas e, assim, aumentando a vazão por meio do paralelismo de consumo.

Um sistema com múltiplas filas de consumo pode gerar assimetrias de distribuição [Fang et al. 2011][Li et al. 2011][Tran et al. 2011], o que leva a um desbalanceamento do sistema ao consumir as mensagens dessas subfilas. Dessa forma, é realizada no middleware IOD a ordenação dos grupos, no momento em que são criadas ou removidas worker threads, iniciando a partir da thread que tem o maior número de mensagens até a que tem o menor número. Em seguida, é realizada a distribuição desses grupos entre as worker threads utilizando o algoritmo round robin. Além disso, em cada rodada de consumo, é realizado o processamento de uma mensagem de cada grupo por meio do FQ (Fair Queueing), que é um algoritmo que permite múltiplas filas de mensagens compartilharem a mesma capacidade de processamento, garantindo justiça no consumo e evitando inanição por conta de fluxos pesados. Além disso, também foi utilizado o algoritmo First Come First Served [Tanenbaum and Woodhull 2007], para preservar a integridade sequencial no consumo das mensagens que pertencem ao mesmo grupo.

Para alcançar a elasticidade no middleware IOD, fez-se necessário realizar a análise da vazão de mensagens na filas, a limitação de criação de worker threads por CPU e por desvio padrão da vazão média de saída por grupo, bem como a recuperação de falhas com elasticidade, as quais serão descritas nas próximas subseções.

\subsection{Análise da Vazão Média de Entrada e Saída}

Durante a rajada de mensagens recebidas, caso uma aplicação distribuída não tenha uma vazão de consumo compatível com a de geração, ocorrem acúmulos nas filas, ou seja, enfileiramentos que geram atrasos no processamento e, consequentemente, redução na qualidade dos serviços prestados. Dessa forma, para obter o número de worker threads que serão necessárias para conter esse enfileiramento na presença de rajadas de mensagens, é necessário calcular a vazão média de entrada e saída, e a média da relação entre as mensagens de entrada e saída. Esses cálculos são realizados a partir do momento da 
detecção de crescimento da fila, aqui denominada Growth Detection $(G D)$, até o Critical Point $(C P)$, que é o momento onde são criadas novas worker threads. O GD ocorre quando a relação entre as mensagens de entrada e de saída torna-se maior do que um, gerando enfileiramento. O GD é detectado pela Equação 1,

$$
\text { GrowthDetection }=\left(\frac{\text { Input }}{\text { Output }}>1\right)
$$

onde, Input é o número de mensagens de entrada e Output é o número de mensagens de saída.

Assim sendo, novas worker threads são criadas no momento em que o acúmulo gerado na fila não pode ser tratado antes do fim do tempo máximo definido para tratamento das mensagens. Esse momento ocorre no $C P$ e a ação de aumentar worker threads é denominada Scale $U p(S U)$. O CP é encontrado quando o número de mensagens na fila é maior do que o valor dado pela Equação 2,

$$
\text { MaxQueueSize }=(\operatorname{avgTOUT} \times(\operatorname{schTIME}-(h N O W-h S T A R T)))
$$

onde, a variável avgTOUT é a vazão média de saída; schTIME é o tempo máximo para o tratamento de mensagens na fila; $h N O W$ é o tempo atual; $h S T A R T$ é o tempo de início, onde o valor dado pela Equação 1 se torna verdadeiro.

O Scale Down (SD) ocorre no Exit Point, que é o momento onde as worker threads são removidas. Ele é obtido pela Equação 3,

$$
\text { QueueSize }<\left(\frac{\operatorname{avgTIN}}{2}\right)
$$

onde, QueueSize é o tamanho atual da fila, e avgTIN é a vazão média de entrada desde o $C P$. A divisão de avgTIN pela metade destina-se a evitar o problema da detecção do limiar de elasticidade para baixo [Imai et al. 2012], o que faz com que a fila de mensagens tenha uma nova tendência de crescimento. Ele foi inspirado pelo decremento multiplicativo utilizado em algoritmos de congestionamento do TCP (Transmission Control Protocol), em que, quando há um timeout, o limiar é definido como a metade da janela de congestionamento atual [Tanenbaum and Wetherall 2010].

Em adição às Contention Threads $(C T)$, que são as worker threads de contenção de rajadas, um outro conjunto de worker threads é necessário, o qual é chamado de Zero Threads $(Z T)$, para consumir as mensagens que se acumularam na fila desde o $G D$ até o $C P$. Dessa forma, são criadas várias worker threads, do tipo $Z T$ ou $C T$, sendo que cada uma delas está associada a várias subfilas de mensagens separadas por grupos, para que ocorra o aumento da vazão por meio do paralelismo de consumo.

\subsection{Análise do Consumo de CPU}

Conhecer o consumo médio de CPU por worker thread é útil para determinar limites para o $S U$ (Scale Up). Assim, ao detectar rajadas de mensagens de entrada, pode ser iniciado o processo para a medição de consumo médio de CPU, por worker thread e por nó do cluster, para determinar se a vazão necessária para conter o crescimento, e zerar a fila pode ser atendido, sem a saturação de uso de CPU do servidor. 


\subsection{Análise do Desvio Padrão da Vazão Média de Saída por Grupo}

As rajadas de mensagens de entrada podem ser originadas a partir de vários grupos. Para atender as rajadas de $n$ grupos, o middleware IOD decide criar várias worker threads, de acordo a vazão média de saída medida durante o crescimento da fila, como descrito na análise da vazão média de entrada e saída, apresentada na Seção 3.2.

No entanto, quando as rajadas são originadas a partir de um pequeno grupo de mensagens, criar várias worker threads pode significar um desperdício de recursos e, dependendo da vazão de consumo, pode não ser suficiente para conter o crescimento da fila e ainda gerar $S U$ ( Scale $U p$ ) recursivo, sem resolver o problema de crescimento da fila. Para resolver esse problema, antes do $S U$ (Scale $U p$ ) e durante a rajada de mensagens, é realizada a medição da vazão média de saída por grupo, que será utilizada no cálculo do desvio padrão no momento de realizar o $S U$. Uma variável aleatória em uma distribuição normal tem $95 \%$ de chance de estar a menos de dois desvios-padrão de sua média [Downing and Clark 2011]. Assim, como a vazão média de saída foi normalizada, em no máximo uma mensagem de cada grupo por rodada por meio do algoritmo FQ, após $n$ rodadas, quando a média da vazão de saída se distancia muito do desvio padrão, ou seja, mais de duas vezes, é possível detectar rajadas vindas de grupos específicos. Dessa forma, é criada uma quantidade de worker threads limitada pelo número de grupos, cuja vazão média de saída seja maior do que duas vezes o desvio padrão identificado durante a rajada de mensagens. Essa abordagem tem o objetivo de criar worker threads para o tratamento específico dos grupos de mensagens nos quais as rajadas foram identificadas.

\subsection{Tolerância a Falhas}

No middleware IOD foi utilizado o suporte às características de tolerância a falhas de software por omissão do processo, não sendo consideradas as falhas de hardware. Assim, as mensagens das filas continuam a ser processadas a partir do ponto em que ocorreu a falha, sem a necessidade de uso da replicação de processos.

Para suportar os mecanismos de recuperação rápida de falhas [Bicer et al. 2010] [Castro et al. 2012] [He et al. 2012] [Wang et al. 2009], se um processo falha e o número de mensagens na fila é maior do que o CP (Critical Point), mecanismos de elasticidade no middleware são necessários para realizar o $S U$ (Scale Up). Assim, quando os processos são reiniciados após uma falha, se as rajadas de entrada continuarem, o $S U$ ocorre rapidamente por meio da detecção do $C P$ maior do que o QueueSize. No entanto, nesse momento é necessário um outro mecanismo para proporcionar elasticidade, caso as rajadas tenham terminado após a queda e antes do reinício do processo. Nesta situação, se o número de mensagens na fila estiver acima de avgTOUT x schTIME, as medidas de vazão, antes de decidir realizar o $S U$, são realizadas por somente $10 \%$ do schTIME. Isso é necessário para realizar $S U$ de uma maneira mais rápida, como é o caso quando as rajadas terminam após a queda, e antes do reinício rápido do processo. O mesmo cenário ocorre quando as rajadas de mensagens não param, mesmo após o reinício rápido do processo.

Assim sendo, nota-se que a implementação eficiente de tolerância a falhas em um ambiente distribuído necessita ser realizada em conjunto com um mecanismo de elasticidade, exatamente como proposto pelo middleware IOD. 


\section{Resultados}

Para os testes do middleware IOD foi criado um simulador, onde foram codificadas duas threads: uma produtora que escreve pacotes na fila de mensagens, e outra consumidora que lê a partir da fila e entrega a mensagem para o middleware IOD. Para esses testes, foi utilizado um tempo máximo de tratamento de mensagens de 80 segundos, e a thread produtora foi programada para gerar mensagens a uma taxa cinco vezes maior do que a thread consumidora. $\mathrm{O}$ objetivo dessa configuração é gerar o enfileiramento de mensagens e demonstrar o funcionamento da elasticidade proposta pelo middleware IOD.

Assim sendo, as subseções a seguir descrevem os testes realizados para verificar o aumento e a diminuição dinâmica das worker threads em função da vazão de entrada e saída; o aumento das worker threads com limite em função do consumo de CPU; e a tolerância a falhas com elasticidade.

\subsection{Criação e Remoção Dinâmica de Threads}

Os testes apresentados nesta seção têm o objetivo de verificar o funcionamento da criação e da remoção de worker threads, dinamicamente, em função da vazão média de entrada e de saída de mensagens.

Para isso, foram feitos testes com rajadas de geração de pacotes a cada 10 milisegundos e consumo a cada 50 milisegundos, ou seja, geração de mensagens a uma taxa cinco vezes maior do que o consumo. O objetivo foi testar o comportamento do middleware IOD diante da necessidade de criar dinamicamente threads. Assim sendo, os experimentos mostraram que ocorreu o aumento adequado do número de worker threads para incrementar a vazão de consumo, como mostra a Figura 1, na qual o eixo $x$ corresponde ao tempo decorrido e o eixo y corresponde ao número de mensagens na fila (QueueSize), ao valor da Equação 2 (MaxQueueSize), a vazão média de entrada (avgTIN) ou a vazão média de saída (avgTOUT), dependendo da variável analisada. No cenário apresentado na Figura 1, o CP foi detectado com 1.130 mensagens na fila, aos 14 segundos após o início da rajada de mensagens, como mostrado na Tabela 1. Aos 48 segundos após o $S U$, o algoritmo decide remover cinco threads de forma que a vazão média de saída fique compatível com a vazão média de entrada. Assim, o middleware IOD se mostrou satisfatório quanto à criação e remoção de worker threads em função da vazão, pois ele foi capaz de criar 12 worker threads, e encerrar com o cenário de gargalo da fila em um período de 23 segundos, ou seja, dentro do limite máximo de tratamento de mensagens definido em 80 segundos. O número de mensagens ficou em um nível controlado, pois a estabilização do algoritmo ocorreu com 33 mensagens de entrada e saída, ou seja, um valor pequeno e controlável.

Tabela 1. Novos Tempos do Algoritmo de Elasticidade.

\begin{tabular}{|l|c|c|c|c|c|}
\hline Momento & Duração (segundos) & Entrada & Saída & Threads & Enfileiramento \\
\hline \hline Início & 14 & 99 & 19 & 1 & 0 \\
\hline Scale Up & 9 & 99 & 197 & 12 & 1.130 \\
\hline Scale Down & 57 & 99 & 99 & 7 & 33 \\
\hline
\end{tabular}




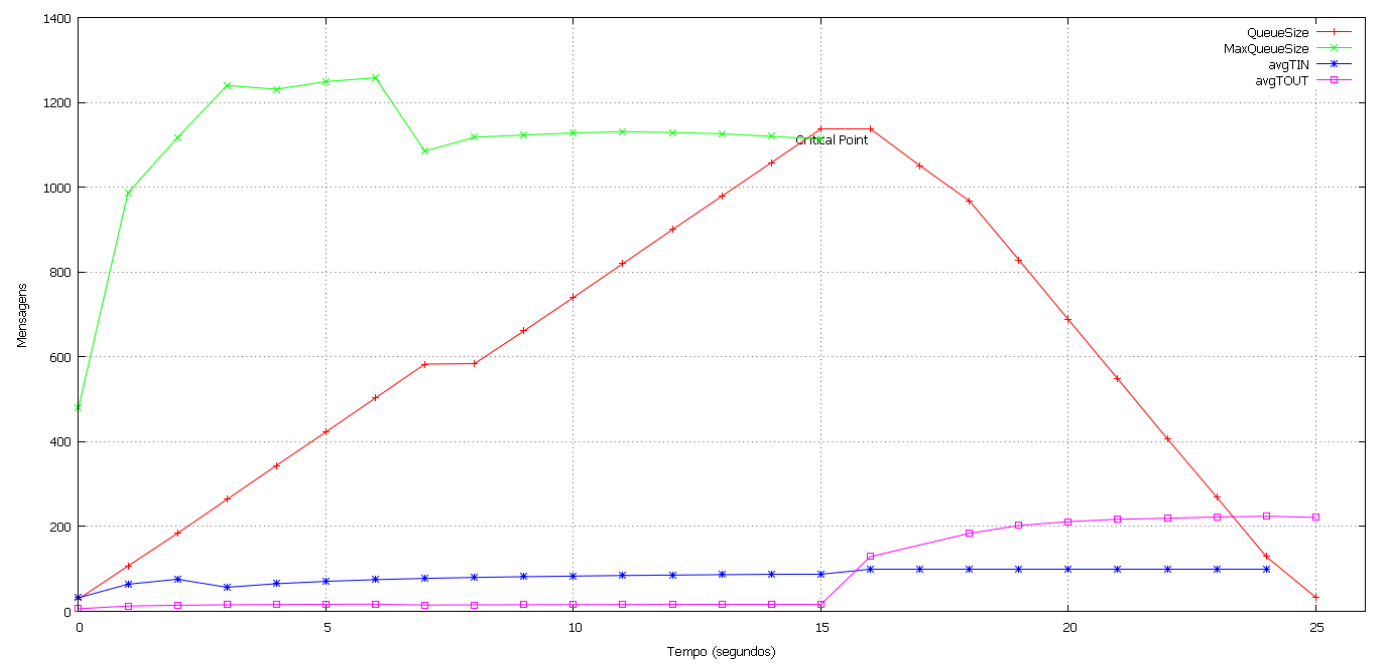

Figura 1. Fila de Msgs com a Criação e a Remoção de Worker Threads.

\subsection{Criação de Threads Limitada pelo Consumo Médio de CPU}

O objetivo deste teste é validar se o algoritmo de elasticidade evita a saturação do consumo de CPU dos servidores, por meio da verificação do comportamento do aumento de worker threads limitado pelo consumo médio de CPU.

Para isso, foi utilizado no simulador a técnica de busywait nas worker threads de consumo, para simular um alto uso de CPU. Os testes foram realizados com o produtor a 10 milisegundos, e com o consumidor a 50 milisegundos, de forma que ocorra o enfileiramento das mensagens. Os resultados da fila de mensagens são mostrados na Figura 2, onde o eixo $x$ corresponde ao tempo decorrido e o eixo $y$ corresponde ao número de mensagens na fila (QueueSize), ao valor da Equação 2 (MaxQueueSize), a vazão média

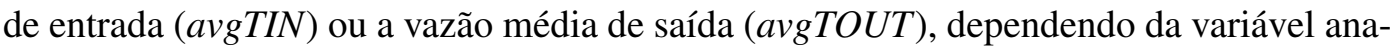
lisada.

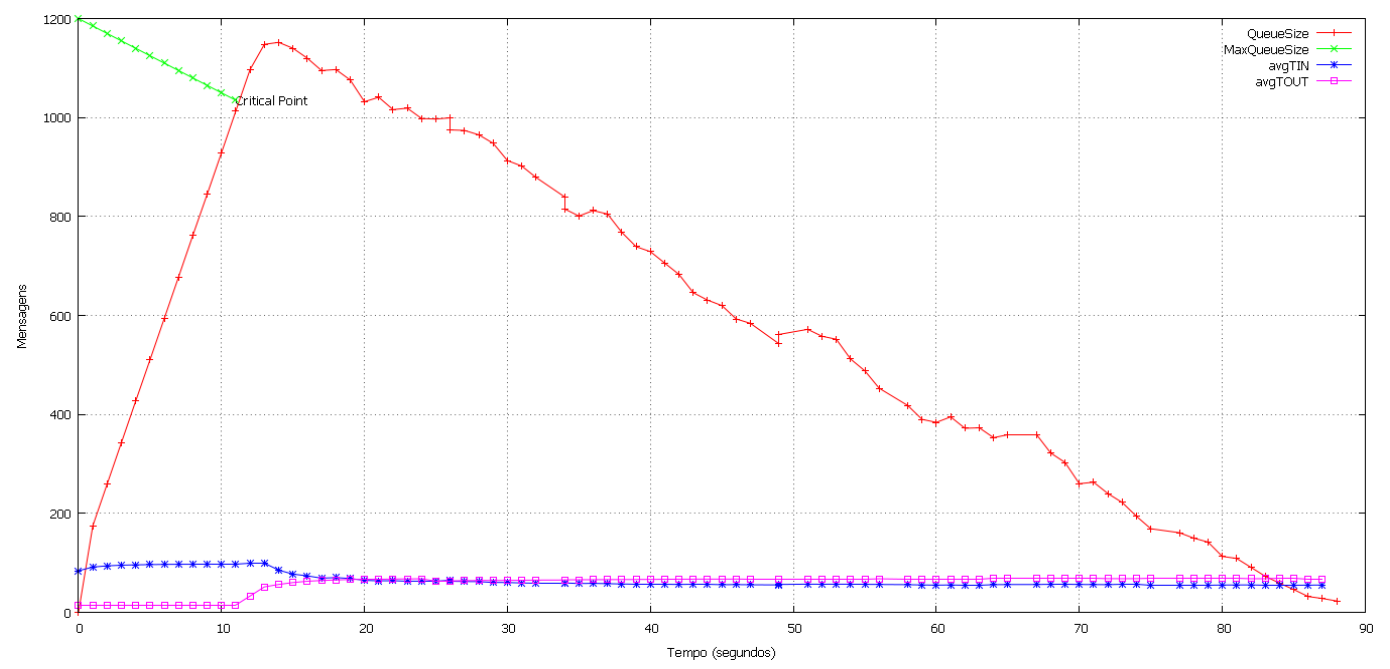

Figura 2. Comportamento da Fila de Mensagens com Limites de CPU. 
Como pode ser observado na Figura 2, a diminuição do número de mensagens ocorre de maneira mais lenta, pois o número de threads é limitado em função do uso de CPU médio das worker threads, de forma que não ultrapasse $100 \%$ do servidor. Além disso, a diminuição não é linear como mostrado na Figura 1, pois não foram criadas todas as worker threads necessárias para que a diminuição das mensagens nas filas ocorresse no tempo requerido.

Tabela 2. Comportamento do Middleware IOD com Limitação de CPU.

\begin{tabular}{|l|c|c|c|}
\hline Momento & Threads Necessárias & Threads Criadas & Threads Removidas \\
\hline \hline Scale Up & 8 & 6 & N/A \\
\hline Scale Down & N/A & N/A & 0 \\
\hline
\end{tabular}

Tabela 3. Comportamento da CPU no Algoritmo de Elasticidade com Limites.

\begin{tabular}{|l|c|c|c|}
\hline Momento & Média por Worker Thread & Total Necessária & Total Residual do Servidor \\
\hline \hline Scale Up & $11.30 \%$ & $90.47 \%$ & $73.89 \%$ \\
\hline ScaleDown & N/A & N/A & N/A \\
\hline
\end{tabular}

De acordo com a Tabela 2, o middleware deveria ter criado oito worker threads, baseado na vazão média de entrada. No entanto, como mostrado na Tabela 3, o consumo médio de CPU por worker thread foi de $11.30 \%$, e ultrapassaria o limite de CPU residual do servidor, que era de $73.89 \%$. Assim, o algoritmo de elasticidade criou apenas seis worker threads (2 a menos), para evitar a saturação do uso de CPU da máquina. No momento de escalar para baixo, a vazão média de saída ficou em 68 mensagens por segundo e, portanto, abaixo da vazão média de entrada que era de 85.33 mensagens por segundo. Dessa forma, o algoritmo de elasticidade não detectou a necessidade de remover worker threads.

Considerar a saturação do ambiente é importante para que a aplicação distribuída seja capaz de identificar dinamicamente os limites de carga que é capaz de processar de acordo com a infraestrutura para a qual foi dimensionada. Dessa forma, o sistema ao identifcar que necessita de mais processamento e não aumentou o número worker threads devido a uma limitação em sua capacidade de processamento, pode gerar alarmes para seja realizado o correto dimensionamento de acordo com a carga exigida.

\subsection{Ativação da Elasticidade após o Reinício Rápido do Processo}

O objetivo deste teste é validar se, após a queda de uma processo durante o tratamento de mensagens, a elasticidade é iniciada sem a presença de rajadas de pacotes, e após o reinício rápido do processo. Dessa forma, foi realizada a simulação da queda do processo e o seu reinício com a fila contendo mensagens com um número acima do $C P$.

Na Figura 3 o eixo $x$ corresponde ao tempo decorrido e o eixo $y$ corresponde ao número de mensagens na fila (QueueSize), ao valor da Equação 2 (MaxQueueSize), a vazão média de entrada (avgTIN) ou a vazão média de saída (avgTOUT), dependendo da variável analisada. Assim, alguns segundos após o reinício do processo, a inclinação do 


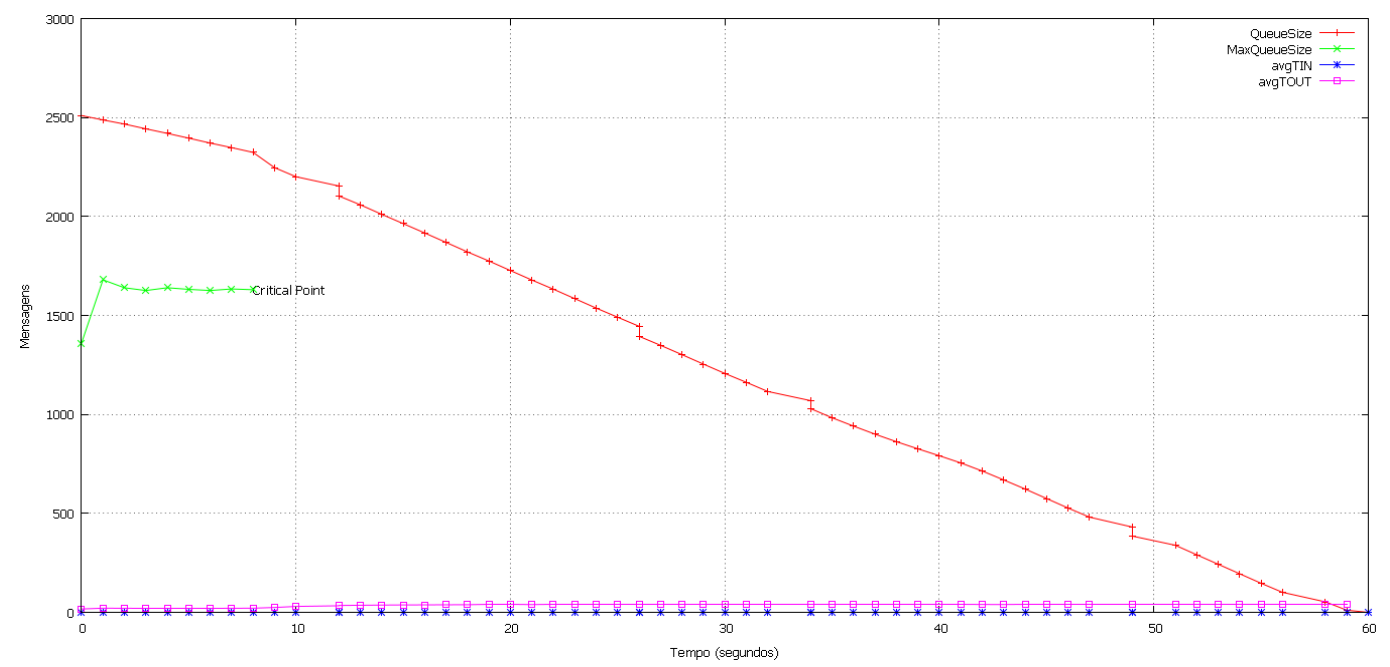

Figura 3. Comportamento da Fila de Mensagens após o Reinício Rápido.

consumo de mensagens aumenta, indicando o incremento do número de worker threads para consumir as mensagens antes do término do tempo máximo de tratamento de mensagens. Este comportamento demonstra que, mesmo com falha no processo, o middleware consegue ser elástico diante de uma demanda gerada anteriormente, e após o reinício rápido do processo.

Assim sendo, diante dos testes realizados, foi possível notar que o middleware IOD proposto é capaz de implementar elasticidade em uma plataforma distribuída. Além disso, o uso de filas de mensagens por processo, mostrou-se um método tolerante a falhas, pois a queda de um processo não impacta em funcionalidades de outros processos [Wang et al. 2009][Castro et al. 2012]. Assim, escrever em filas diferentes com funcionalidades distintas, paraleliza o tratamento, escala o sistema e torna o processamento mais rápido.

Outra importante contribuição foi utilizar o paralelismo de consumo em filas de mensagens, por meio do agrupamento de mensagens correlacionadas em subfilas, pois ele proporcionou a diminuição dos custos com a infraestrutura necessária para manipular as filas de mensagens. Dessa forma, criar mecanismos que proveem o aumento da vazão de consumo, melhoram significativamente a qualidade dos serviços prestados, uma vez que a mesma quantidade de massa de dados pode ser processada em menos tempo e por uma quantidade menor de servidores.

\section{Conclusões e Trabalhos Futuros}

O custo cada vez mais alto de equipamentos de infraestrutura mostra que é essencial utilizar os recursos disponíveis de maneira otimizada e eficiente. Dessa forma, o dimensionamento dinâmico de recursos mais próximo da quantidade de processamento necessária para prover serviços, mostra-se cada vez mais importante, pois significa economia de custos sem perda da qualidade dos serviços prestados. Assim, o middleware IOD mostrou ser possível alcançar os requisitos de elasticidade, adaptando-se as condições da carga de processamento, aumentando ou diminuindo o número de worker threads de acordo com a demanda requerida. Além disso, o middleware IOD mostrou como otimizar o uso de 
recursos de TI por meio do paralelismo de tarefas, usando múltiplas worker threads para processar as filas de mensagens.

Uma contribuição alcançada neste artigo foi a criação das Equações 2 e 3 para determinar os pontos críticos de entrada e saída para o tratamento paralelo das filas de mensagens, dinamicamente, de acordo com a vazão de entrada e de saída e a detecção de crescimento dada pela Equação 1. Estas equações são baseadas no comportamento dinâmico de cada servidor e nos limites de tempo impostos para o processamento de mensagens, os quais estão diretamente relacionados com os parâmetros de QoS (Quality of Service) definidos com cada cliente da aplicação distribuída que utiliza fila de mensagens. Todavia, uma limitação atual do middleware IOD está ligada ao fato de não ser possível paralelizar o tratamento de mensagens associadas ao mesmo grupo, por conta da restrição de integridade sequencial que impõe ordenação no processamento.

Assim sendo, entre os trabalhos futuros estão os estudos de pipelining para a execução paralela de instruções que podem ser exploradas para o adiantamento do processamento de mensagens associadas ao mesmo grupo, sem a geração de conflito de integridade sequencial.

\section{Referências}

Abbes, H., Cerin, C., Jemni, M., and Walid.Saad (2010). Fault tolerance based on the publish-subscribe paradigm for the bonjourgrid middleware. 11th IEEE/ACM International Conference on Grid Computing, pages 57-64.

Bicer, T., Jiang, W., and Agrawal, G. (2010). Supporting fault tolerance in a data-intensive computing middleware. 2010 IEEE International Symposium on Parallel \& Distributed Processing (IPDPS), pages 1-12.

Castro, M., Rexachs, D., and Luque, E. (2012). Transparent fault tolerance middleware at user level. 2012 International Conference on High Performance Computing and Simulation (HPCS), pages 566-572.

Coulouris, G., Dollimore, J., and Kindberg, T. (2009). Distributed Systems Concepts and Design. Editora Pearson Prentice Hall, fourth edition.

Downing, D. and Clark, J. (2011). Estatİstica Aplicada. Editora Saraiva, third edition.

Fang, W., Jin, B., Zhang, B., Yang, Y., and Qin, Z. (2011). Design and evaluation of a pub/sub service in the cloud. International Conference on Cloud and Service Computing, pages 32-39.

Gray, J. S. (2003). Interprocess Communications in LinuxA: The Nooks Crannies. Editora Pearson Prentice Hall, first edition.

Guo, Y., Hanm, R., Satzger, B., and Truong, H.-L. (2012). Programming directives for elastic computing. Internet Computing and IEEE, pages 72-77.

He, C., Weitzel, D., Swanson, D., and Lu, Y. (2012). Hog: Distributed hadoop mapreduce on the grid. SC Companion: High Performance Computing and Networking Storage and Analysis, pages 1276-1283.

Herbst, N. R., Kounev, S., and Reussner, R. (2013). Elasticity in cloud computing: What it is, and what it is not. In Proceedings of the 10th International Conference on Autonomic Computing (ICAC 13), pages 23-27, San Jose, CA. USENIX. 
Imai, S., Chestna, T., and Varela, C. A. (2012). Elastic scalable cloud computing using application-level migration. IEEE/ACM Fifth International Conference on Utility and Cloud Computing, pages 91-98.

Leitner, P., Inzinger, C., Hummer, W., Satzger, B., and Dustdar, S. (2012). Applicationlevel performance monitoring of cloud services based on the complex event processing paradigm. 5th IEEE International Conference on Service-Oriented Computing and Applications, pages 1-8.

Li, M., Ye, F., Kim, M., Chen, H., and Lei, H. (2011). A scalable and elastic publish/subscribe service. IEEE International Parallel \& Distributed Processing Symposium, pages $1254-1265$.

Ma, R. K., Lam, K. T., Wang, C.-L., and Zhang, C. (2010). A stack-on-demand execution model for elastic computing. 39th International Conference on Parallel Processing, pages 208-217.

Marshall, P., Tufo, H., and Keahey, K. (2012). Provisioning policies for elastic computing environments. 26th International Parallel and Distributed Processing Symposium Workshops \& PhD Forums, pages 1085-1094.

Martins, R., Narasimhan, P., Lopes, L., and Silva, F. (2010). Lightweight fault-tolerance for peer-to-peer middleware. 29th IEEE International Symposium on Reliable Distributed Systems, pages 313-317.

Perez, J., Germain-Renaud, C., Kégl, B., and Loomis, C. (2009). Responsive elastic computing. ACM/IEEE Conference on International Conference on Autonomic Computing, pages 55-64.

Smith, J. M. (1986). A survey of software fault tolerance techniques. pages 165-170.

Sugiki, A. and Kato, K. (2011). An extensible cloud platform inspired by operating systems. Fourth IEEE International Conference on Utility and Cloud Computing, pages 306-311.

Tanenbaum, A. S. and Steen, M. V. (2008). Distributed systems: principles and paradigms. Editora Pearson Prentice Hall, second edition.

Tanenbaum, A. S. and Wetherall, D. J. (2010). Computer Networks. Editora Pearson Prentice Hall, fifth edition.

Tanenbaum, A. S. and Woodhull, A. S. (2007). Operating Systems Design and Implementation. Editora Pearson Prentice Hall, third edition.

Tran, N.-L., Skhiri, S., and Zimányi, E. (2011). Eqs: an elastic and scalable message queue for the cloud. Third IEEE International Conference on Cloud Computing Technology and Science, pages 391-398.

Wang, J., Jian-Wen Chen, Y. D., and Zheng, D. (2009). Research of the middleware based fault tolerance for the complex distributed simulation applications. International Conference on Computational Intelligence and Software Engineering (CiSE), pages 14. 